\title{
Intelligent Decision Support System Development Technology of Automotive Mechanical System
}

\author{
Zongqi Yuan ${ }^{1,}$ a * \\ ${ }^{1}$ Guizhou Polytechnic College of Communictions, Guiyang, Guizhou 550000,China \\ aemail: zongqi_yuan@163.com
}

\begin{abstract}
Keywords: Decision support system; Artificial intelligence; Case-based reasoning; Reasoning rules;
\end{abstract} Intelligent design.

\begin{abstract}
Morphological matrix method and its key legal point of view the main function of the program from portfolio analysis, and then the combination of a good program to list, due to the combination of a lot of the number of programs, and therefore difficult to compare them one by one evaluation, it is difficult to get the best program. The main problem lies in the expert system knowledge acquisition and reasoning more difficult. Intelligent design of the car's mechanical system is actually an instance of reasoning and matching process. In this paper, automotive Intelligent Design System Based on CBR and RBR in an attempt to improve the efficiency of product design. ICAD system technology and artificial intelligence expert system optimization design, finite element analysis, computer graphics and other various numerical calculation methods combine their respective merits, complement each other, the aim is to make the computer as much as possible to participate in decision - making, structural design, performance analysis, graphics and so the whole process.
\end{abstract}

\section{Introduction}

According to the results of various design programs, experts from the mind of the index database, to find a suitable evaluation system, using integrated mathematical model of comprehensive evaluation of each viable options from which to choose the best option, then the need to apply fuzzy mathematics and systems engineering theory and methods [1-3]. If all feasible options are difficult to accept, we will have to re-design and re-analysis, evaluation, decision making, ad infinitum, until the best design [4]. The most obvious feature ICAD is to have the knowledge base to solve the problem, have the knowledge to select and coordinate the project database and graphics libraries and other resources together to complete the design task of reasoning decision-making mechanism [5, 6]. Therefore, ICAD system in addition to the engineering database, graphics libraries CAD features, it should also have knowledge, reasoning and other intelligence module [7].

After determining the functional characteristics of the car, the designers determine the form of the engine based on past experience in the design and design examples, drive, drive train form, suspension form, body form, in the form of steering system, brake system and other forms, the stage is determined the composition of the structure of an automobile, and the design is divided into the engine selection, powertrain design, suspension system design, design brake system, steering system and other sub-design problems [8-10].

Directly determines the system's problem-solving capabilities, including knowledge base, model base, methods and databases and their management systems. Knowledge is knowledge in its broadest sense, to use it to store the system design experience, design knowledge, design specifications and design examples. Model Stock put two models, namely reasoning and mathematical models, the former is the $\mathrm{Al}$ inference engine, including CBR inference engine, forward chaining, backward reasoning machine; the latter algorithm, optimization, decision analysis, mathematical plan model and model design. The method is actually stored in the database is a computer algorithm model.

\section{The overall structure of an automobile intelligent design system}


Cars intelligent design system by constructing the case base knowledge can be applied to maximize the long-term accumulation of experience designer, conceptual design to reduce duplication of effort and improve the efficiency of design and development of automotive mechanical system and shorten development time. According to auto mechanics problems encountered in system development (such as knowledge matching workload), build cars and auxiliary machinery case base knowledge repository of key components and overall performance automobile engine, radiator, and other gear for analysis. The automobiles intelligent design system shown in Fig. 1. User-friendly user interface, enter the design requirements of the car, completed the overall design of the car selection and function of the mechanical system, complete the structural design and mechanical design parameters of the key components of the car through the process of reasoning, if the design does not meet the design requirements are the result again design.

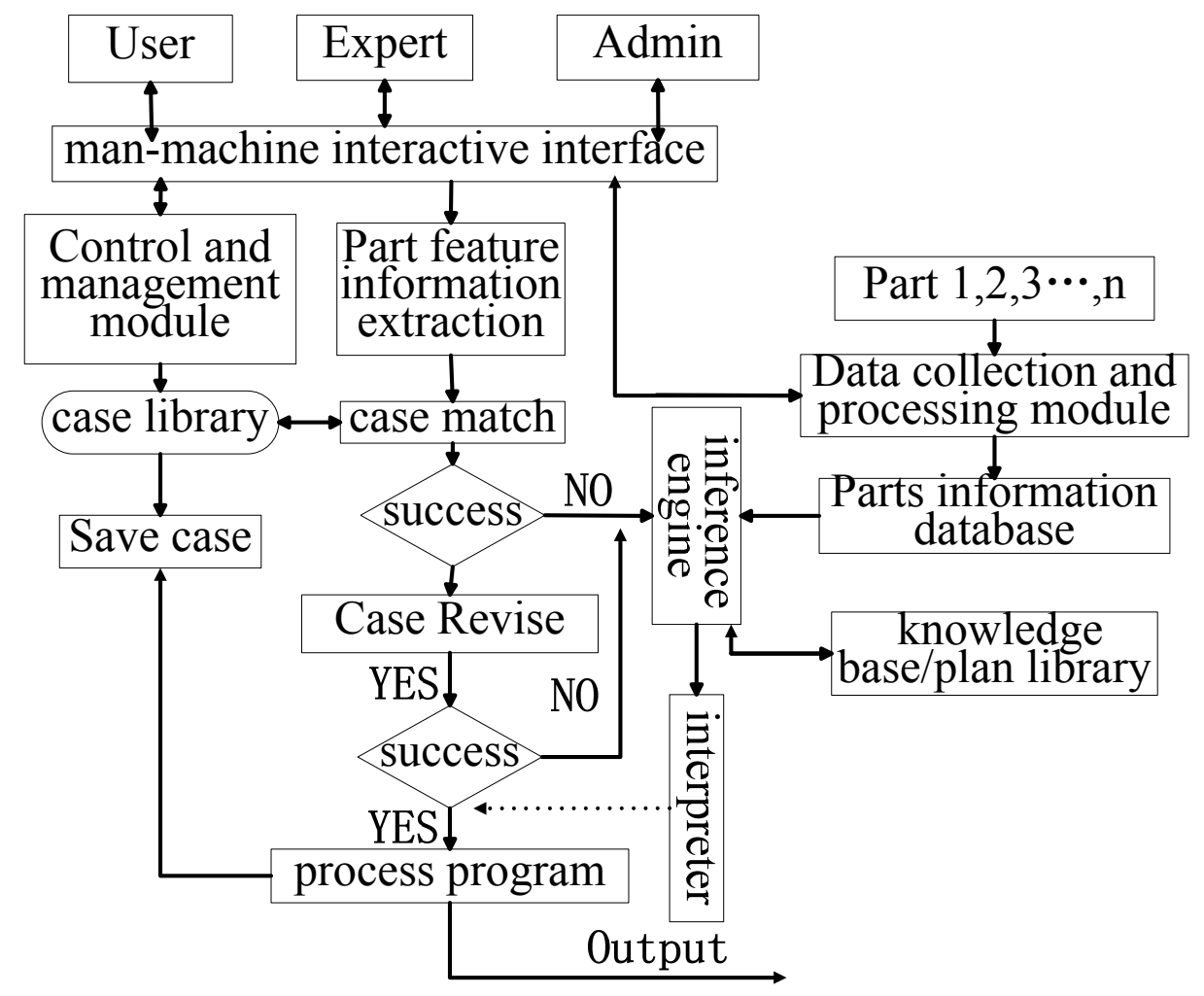

Figure 1. Mechanical design system block diagram smart cars

Models and methods are two sides of the same problem, only. Is the manifestation is different, the former general performance of mathematical equations, which showed Algorithm. From a macro point of view, the model can be seen as a unified model and methods. Especially in the computer, not the main form of the model equations, the algorithm is the main model. The general form of the model equations in text form as documentation model, and the model will be developed into a computer program algorithm to complete the model, to achieve the purpose of solving the model.

\section{Case retrieval algorithm design}

$\mathrm{CDR}$ in the search algorithm is different from the database retrieval request to find an approximate match for instance meet certain requirements based on keywords that match the value retrieved instance. Examples of CBR retrieval is generally divided into the following three steps (Fig. 2):

(1) Identification of the characteristics of the current analysis of the problem, through interaction with the user, extracting relevant features, create an instance of the index.

(2) Preliminary matching nearest neighbor, induction, template retrieval method to find a set of candidate instance from the current issues relating to case library. 
(3) Selection of the best examples of the candidate obtained the preliminary matches to sort, select one or several issues most relevant to the current instance.

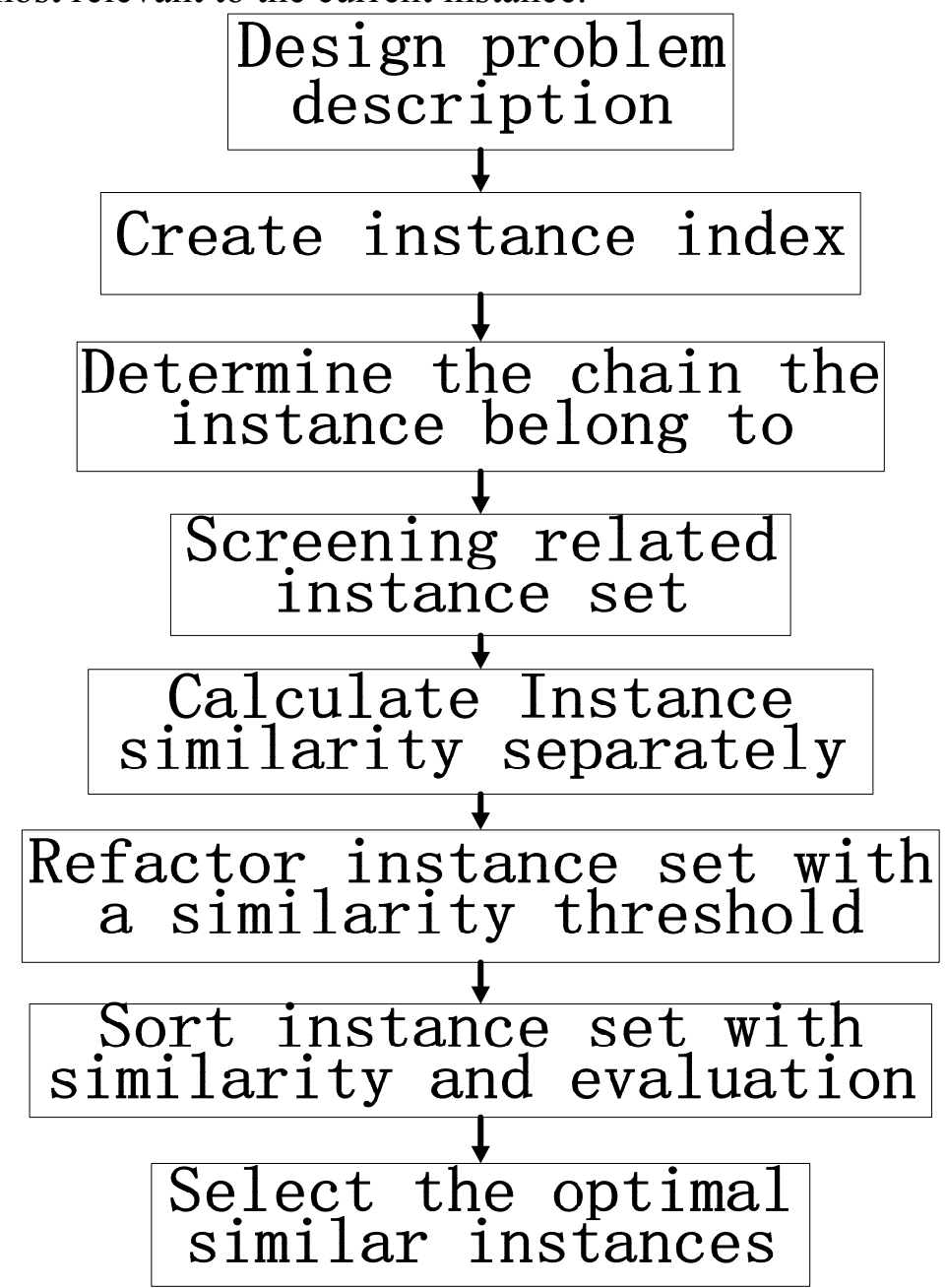

Figure 2. Examples of search algorithm

The above algorithm using similarity between instances of weight calculation Neighbor, which is calculated as:

$$
\mathrm{S}=1-\sqrt{\sum_{i=1}^{n}\left(w_{i}\left(u_{i}^{0}-u_{i}\right) /\left(u_{i}^{1}-u_{i}^{2}\right)\right)^{2}}
$$

Wherein n-an amount of the number of features;

Ui-First a target value of the quantitative characteristics of the design;

Wi-Examples of value calculation for the current $j$-th quantitative characteristics;

VIP prototype system based on the algorithm used in the design of the search program instance, with the use of variable depth Prolog language preference and self-contained or tree blind search function, you can easily retrieve and flexible design of similar instances.

\section{Results and discussion}

Now automotive system designers through the design of intelligent mechanical systems relevant literature, and design and industry experts to exchange knowledge to determine the scope of knowledge and knowledge expression, knowledge of simple, accurate calls. The system is based on a top-down approach and the way the body of the car body of knowledge modeling mechanical systems, first clear product categories, component class, class member, part class and its classification system auto mechanics, auto mechanics then describe system related entities, attributes, relationships yuan body. 
Case library covers a lot of product information automobile mechanical parts, including product structure and function of specific parameters, model, power and uses. For different cases, depending on the attribute parameter can be used to classify the group technology management. The case base is built, to *. dat file format for storage, storage type "case each element name + space + number", to facilitate matching retrieval system operation and extract the parameters for the call. Since many types of automotive mechanical parts, one library does not discuss the case, it is to car clamp unit case base structure described as an example the case of the library, built cars gripper unit case base shown in Fig. 3.

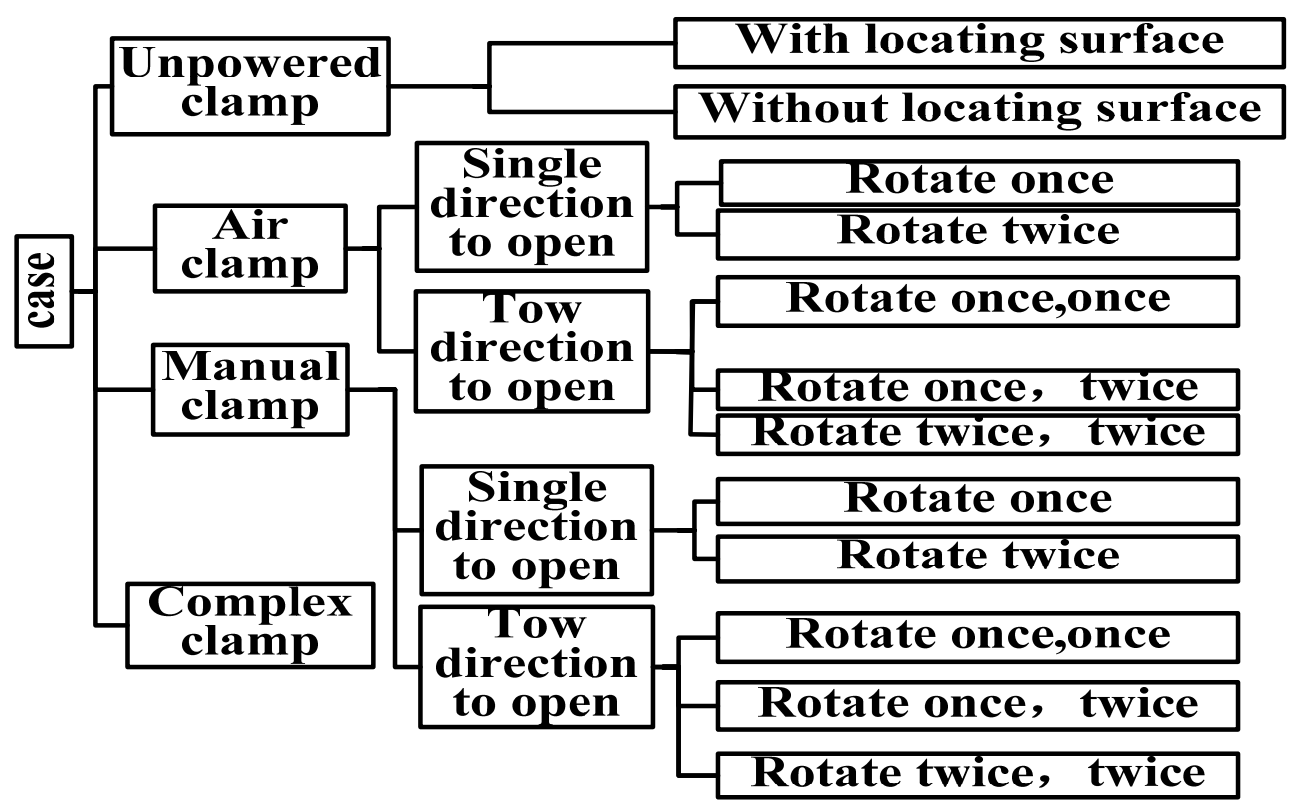

Figure 3. Cars case base

Example positioning of parts mainly refers to the position of unit cases in the automotive mechanical system. When the designer by searching the case or components transferred stations were - assemblies, mounting location is critical to determine whether the correct, you need to coordinate and auto body unit case machine coordinate conversion in the process. Therefore, how to properly transform auto body and mechanical coordinate unit case is the problem to be solved. This article is divided into two steps to transform the coordinates: 1) to coordinate pan, and it coincides with the new coordinate origin; 2) rotational transformation, and achieve axes coincide.

\section{Conclusions}

The database can not only provide the basic data to support decision-making process of mechanical parts, but also for the knowledge base, rule base and case base to provide the underlying data source. In this paper, in the form of knowledge and examples exist of expression, mechanical design knowledge database. This design of automotive intelligent design system, hybrid reasoning process of CBR and RBR car mechanical systems design, application prospects. In future research will further improve the knowledge base and case database auto intelligent design system for users to provide more to meet the needs of automotive mechanical systems knowledge, in order to better complete the design of the car mechanical system to get a more accurate retrieve an instance.

\section{References}

[1] Ali B A A, Sapuan S M, Zainudin E S, et al. Implementation of the expert decision system for environmental assessment in composite materials selection for automotive components[J]. Journal of Cleaner Production, 2015.

[2] Babuska R, Verbruggon H B, Bancan H J L. Fuzzy modeling of enzymatic penicillin-G Anversion. Engineering Application of Artificial Intelligence, 1999, 12 (1): 79-72. 
[3] Xiao W, Weidemann B. Fuzzy modeling and its application to magnetic bearing systems. Fuzzy Sets and Systems, 1995, 73(20): 20-217.

[4] Lee Chang Jun, Prasad Binay, Lee Jong Min. Stochastic nonlinear optimization for robust design of catalysts. Industrial and Engineering Chemistry Research, 2011, 50 (7): 3938-394.

[5] Y. Zhao, W. Wei, X.J. Tang, M.L. Zhao, Choice of Optimized Scheme Parameters of Neural Networks Based on Error Handling, AISS. 17(2012)76-82.

[6] Ho H P. The Influence of Braking System Component Design Parameters on Pedal Force and Displacement Characteristics. Simulation of a passenger car brake system, focusing on the prediction of brake pedal force and displacement based on the system components and their design characteristics[D]. University of Bradford, 2015.

[7] Diakaki C, Papageorgiou M, Papamichail I, et al. Overview and analysis of Vehicle Automation and Communication Systems from a motorway traffic management perspective[J]. Transportation Research Part A: Policy and Practice, 2015, 75: 147-165.

[8] Wang B, Huang X, Wang J, et al. A robust wheel slip ratio control design combining hydraulic and regenerative braking systems for in-wheel-motors-driven electric Vehicles[J]. Journal of the Franklin Institute, 2015, 352(2): 577-602.

[9] Reaidy P J, Gunasekaran A, Spalanzani A. Bottom-up approach based on internet of things for order fulfillment in a collaborative warehousing environment[J]. International Journal of Production Economics, 2015, 159: 29-40.

[10]Gonzalez E D R S, Sarkis J, Huisingh D, et al. Making real progress toward more sustainable societies using decision support models and tools: Introduction to the special volume[J]. Journal of Cleaner Production, 2015. 